Abstract

\title{
Knowledge of Low Energy Availability, Relative Energy Deficiency in Sport and the Female Athlete Triad Amongst Female Athletes and Active Individuals ${ }^{\dagger}$
}

\author{
Jade Winter *, Katherine Black and Rachel Brown \\ Department of Human Nutrition, University of Otago, Dunedin 9054, New Zealand; \\ katherine.black@otago.ac.nz (K.B.); rachel.brown@otago.ac.nz (R.B.) \\ * Correspondence: jade.winter01@gmail.com ; Tel.: +64-277264127 \\ + Presented at the 2019 Annual Meeting of the Nutrition Society of New Zealand, Napier, New Zealand, \\ 28-29 November 2019.
}

Published: 16 December 2019

Background: Low Energy Availability (LEA), Relative Energy Deficiency in Sport (RED-S) and the Female Athlete Triad (Triad) are generally related to the negative health effects of consuming insufficient energy to meet exercise energy expenditure and maintain normal physiological function. In New Zealand (NZ), about 50\% of active women are at risk of LEA. Limited overseas research suggests awareness and knowledge of LEA, Triad and RED-S is low. Awareness of the signs and symptoms of these conditions is important for prevention and early intervention. The aim of this study was to investigate the level of awareness and knowledge of LEA, the Triad and RED-S amongst female athletes and active women living in NZ.

Design: In total 180 physically active females aged 18-45 years completed an online questionnaire consisting of 89 questions derived from previously validated questionnaires.

Results: Overall, 35\% ( $n=60)$ of participants had heard of at least one of the three conditions. Highest level of knowledge was demonstrated for the Triad $(13.5 \%, n=23)$, whilst $5.3 \%(n=9)$ could describe the terms LEA and/or RED-S, and 2.9\% $(n=5)$ had good knowledge of all three conditions. Knowledge of health and performance consequences of consuming insufficient energy was generally very good, although there were clear knowledge gaps in relation to the age at which bone mineral density is attained, and associations between fractures and menstrual dysfunction. Eighty-five percent of participants indicated a desire to learn more about female health.

Conclusion: This study provides valuable insight into the awareness and knowledge of LEA, the Triad and RED-S amongst active women in NZ. Considering the knowledge gaps and willingness to learn more, the findings emphasise the need for education interventions to prevent and detect signs and symptoms of LEA before negative health and performance effects are experienced.

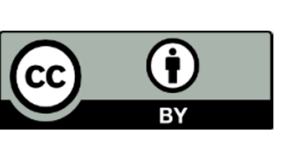

(C) 2019 by the authors. Licensee MDPI, Basel, Switzerland. This article is an open access article distributed under the terms and conditions of the Creative Commons Attribution (CC BY) license (http://creativecommons.org/licenses/by/4.0/). 